Proceedings

\title{
Integration of Microresonant Sensor into a Microfluidic Platform for the Real Time Analysis of Platelets-Collagen Interaction in Flow Condition ${ }^{+}$
}

\author{
Aleksandr Oseev, Benoît Le Roy de Boiseaumarié, Fabien Remy-Martin, \\ Jean-François Manceau, Alain Rouleau, Franck Chollet, Wilfrid Boireau and Thérèse Leblois * \\ FEMTO-ST Institute, CNRS UMR-6174, Université de Bourgogne Franche-Comté, 15B, Av des \\ Montboucons, 25030 Besançon, France; aleksandr.oseev@femto-st.fr (A.O.); benoit.leroy@femto-st.fr \\ (B.L.R.d.B.); fabien.remy@femto-st.fr (F.R.-M.); jfmanceau@femto-st.fr (J.-F.M.); alain.rouleau@femto-st.fr (A.R.); \\ franck.chollet@femto-st.fr (F.C.); wilfrid.boireau@femto-st.fr (W.B.) \\ * Correspondence: therese.leblois@femto-st.fr; Tel.: +33-363-082-456 \\ † Presented at the Eurosensors 2018 Conference, Graz, Austria, 9-12 September 2018.
}

Published: 10 December 2018

\begin{abstract}
The contribution focuses on the development of microresonant sensor solution integrated in microfluidic platform for the haemostasis assessment at realistic rheological flow conditions similar to the one in blood vessels. A multi-parameter sensor performs real time analysis of interactions between immobilized collagen and platelets. The detection and characterization of such interactions at controlled flow rates provide information to evaluate the dynamic of each step of primary haemostasis. The microresonant sensor concept was developed and is described in the contribution.
\end{abstract}

Keywords: microfluidic sensor; biosensor; micro-acoustic sensor; haemostasis

\section{Introduction}

Monitoring of blood ability to clot properly is an important and demanding task in every day clinical practice. Haemostasis is a complex process of the body that stops the bleeding of injured vessels. It involves a coordinated effort between platelets and numerous blood clotting proteins, resulting in the formation of a blood clot and subsequent stopping of the bleed. Its initial step is primary haemostasis which results in a platelet plug and precedes the formation of blood clot closing the vascular lesions. This process requires a precise equilibrium and any deviation can lead to haemorrhages on the one-side or platelet deposits and thrombosis on the other side. A number of parameters should be controlled in order to monitor the haemostasis such as: circulating platelets, blood clotting proteins and the state of vascular walls [1]. Most of the currently available devices that are adapted for clinical practice have several limitations: they do not reproduce rheological conditions of blood; the reproducibility of measurements is insufficient; and a substantial amount of blood is required [2]. The motivation for this work is to improve the pathology evaluation with compact integrated sensor solution in order to manage patients with bleeding risk or under antiplatelet therapy.

The general concept of the integrated microresonant sensor platform is shown in Figure 1. The microfluidic part is designed to replicate the rheological flow conditions of the whole blood in vessel. Locally functionalized interface exposes the flowing whole blood to specific collagen that mimics the blood vessel damage. The microresonant sensor is integrated in a microfluidic structure to complete in-flow monitoring of the collagen-platelets interactions at the functionalized 
biointerface. The microresonant sensor is designed as a bulk acoustic wave resonator with intended coupling to shear bulk acoustic mode (shear excited structure mode is shown in Figure 1) [3]. The sensor is integrated in a microfluidic transparent structure that enables simultaneous analysis of collagen-platelets interactions with acoustical methods and provides an opportunity for optical monitoring (such as fluorescent detection of marked cells). The detection and characterization of such interactions will provide information on each step of primary haemostasis and help determine the overall impact of all blood components involved in the process.

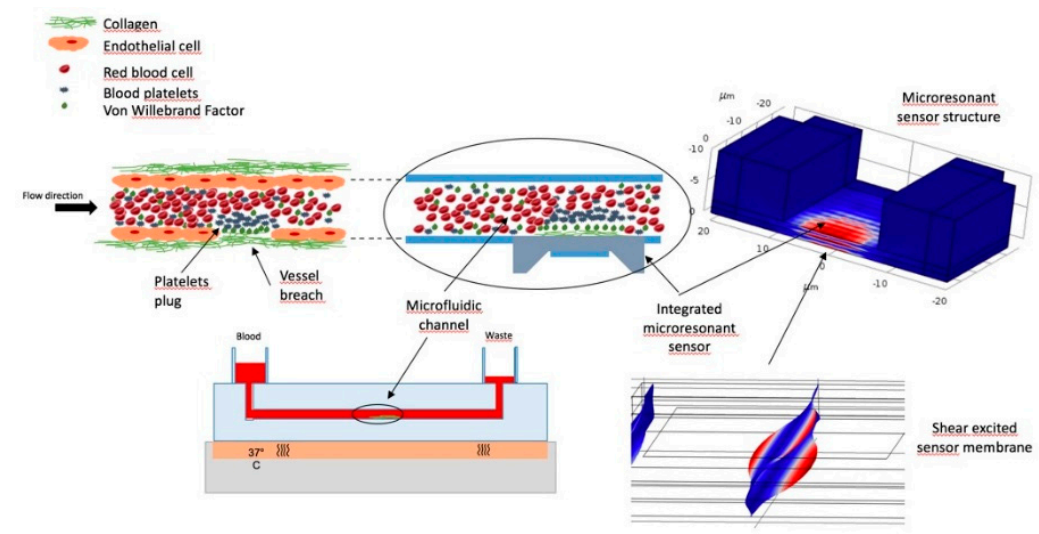

Figure 1. Schematic representation of the sensor platform for the analysis of primary haemostasis integrating the microfluidic system mimicking the rheological blood flow conditions, functionalized interface for the localized platelets binding, integrated microacoustic sensor, flow rate and pressure control system. Displacement field distribution of microresonant structure was obtained using COMSOL Multiphysics ${ }^{\circledR}$ software.

\section{Materials and Methods}

The sensor experimental verification included the experiments conducted with the whole blood, platelets poor plasma (PPP) and platelets rich plasma (PRP). Solution of $10 \mu \mathrm{M}$ fluorochrome DIOC6(3) was incubated for $15 \mathrm{~min}$ at room temperature in the PRP and the whole blood samples to stain the cells. The blood used for the preparation of the samples was collected in $3 \mathrm{~mL}$ blood collection tubes with $75 \mu \mathrm{M}$ PPACK as anticoagulant.

Two different sensor biointerfaces were utilized to study the platelets binding. The sensor with antibody immobilized interface was used for initial studies to ensure platelets single layer formation at the surface of the sensor biointerface. For that reason, antibody anti-CD41 was used at a concentration of $200 \mu \mathrm{g} / \mathrm{mL}$ diluted in a sodium acetate buffer $10 \mathrm{mM}$ (pH4). A sensor with collagen immobilized surface was produced, in order to study the platelets-collagen interaction. We used the collagen Horm $^{\circledR}$ at a concentration of $50 \mu \mathrm{g} \mathrm{mL}^{-1}$.

The sensor gold sputtered surface was functionalized with 11-MUOH and 16-MHA solution overnight. Afterwards, for the antibody immobilization, the sensor was incubated for $30 \mathrm{~min}$ with a mixture of $200 \mathrm{mM}$ EDC and $50 \mathrm{mM}$ sulfo-NHS followed by incubation with anti-CD41 under ultrasonic bath agitation. For the case of collagen immobilized interface, the incubation was completed overnight at room temperature without agitation. Achieved bio-interfaces were blocked with $1 \%$ of RSA in sodium acetate buffer $10 \mathrm{mM} \mathrm{pH} 4.5$ solution to prevent non-specific binding at the sensor surface. For the deactivation of remaining free ester reactive groups, an additional treatment step with ethanolamine $\mathrm{HCl} 1 \mathrm{M} \mathrm{pH} 8.5$ was performed. Afterwards RSA solution was used to passivate the peripheral microfluidic network.

In order to quantify the influence of deposited platelets at the functionalized interface of the resonator, the conventional quartz crystal resonators [4] were utilized in a first approach. As the integrated sensor structure is designed for shear wave, the load from the analyte and from the interface deposited platelets can be quantified with the application of $5 \mathrm{MHz}$ shear bulk acoustic wave resonator with known approximation for introduced load [5,6]. 


\section{Results}

We report initial experimental results where we studied the interactions at the biointerface with shear excited bulk acoustic wave resonator in flow conditions. Experimental response of the sensor with antibody immobilized interface with the whole blood in flow condition (targeting shear rate is $1000 \mathrm{~s}^{-1}$ ) is shown in Figure 2. We recorded the sensor impedance magnitude and phase response during the injection of $500 \mu \mathrm{L}$ of whole blood in the fluidic system. Phosphate buffer saline (PBS) was utilized as running buffer before and after the whole blood injection. As can be seen on the impedance response (Figure 2a,b), introduction of blood significantly changes the magnitude and phase response of the sensor. The rapid change of the sensor response just after the blood injection is explained by significant change of the applied liquid medium (whole blood viscosity and density are significantly different from PBS). The following shift of the sensor resonance towards lower frequency is explained by the evolution of the binding of platelets at the biointerface of the resonator. AFM image of the sensor surface taken upon the completion of the experiment reveals numerous platelets captured at the surface, (Figure 2c).

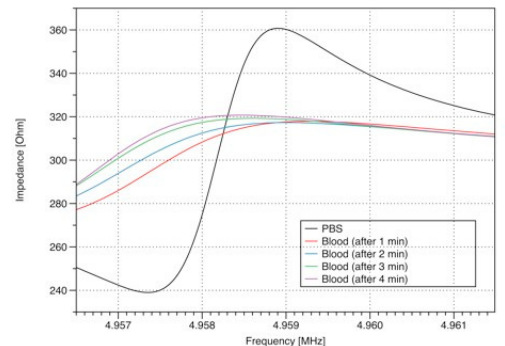

(a)

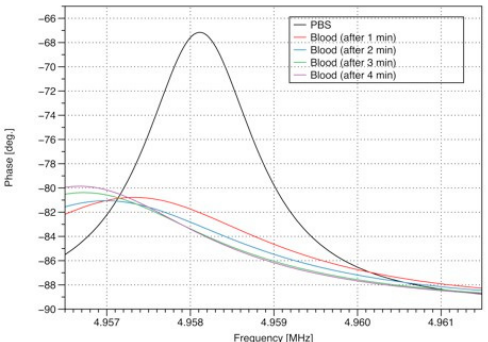

(b)

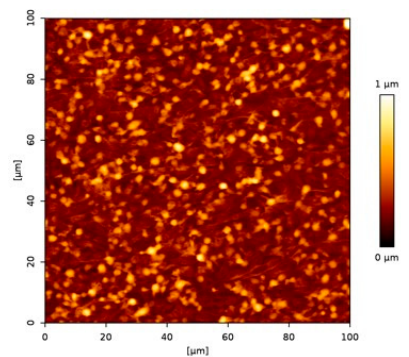

(c)

Figure 2. Antibodies functionalized surface Impedance magnitude (a) and phase (b) response. AFM image of the sensor antibodies bio-functionalized interface with captured platelets (c).

A second experiment was performed with the collagen immobilized sensor interface. In order to highlight the contribution of the blood plasma viscosity/density and assess the limits of the detection of platelets deposited at the surface, experimental investigations were made separately for the platelets poor plasma (PPP) and the platelets rich plasma (PRP). The initial state of the fluidic system provides a response that corresponds to the PBS solution. Subsequent injection of PPP-PBS-PRP-PBS has been done in a manual mode. The control of the state of the sensor biointerface was completed with microscope recording of fluorescence images. In-time recorded variation of the sensor response (phase maximum deviation) is shown in Figure 3a.

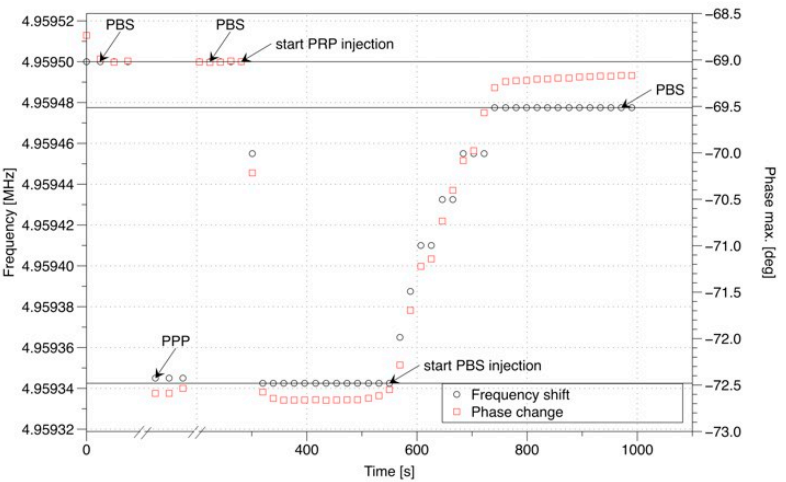

(a)

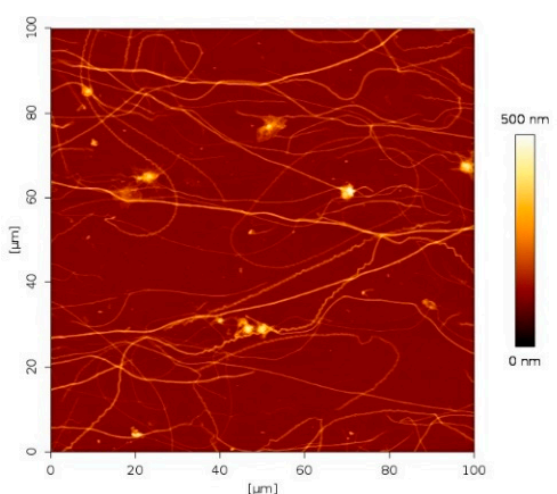

(b)

Figure 3. In-time recording of collagen immobilized sensor response (frequency and magnitude of phase maximum) during PPP/PRP injection experiment (a); AFM image of collagen immobilized biointerface with captured platelets (b). 
As it can be seen, loading of the sensor interface with PPP immediately causes well detectable frequency downshift that is restored when the PBS solution is introduced afterwards. The injection of PRP causes a higher frequency downshift and significant change in frequency of phase maximum in comparison to PPP, the initial state of the resonator is not recovered after continuous injection of PBS solution. The difference corresponds to the acoustic load of the resonator with deposited platelets at the collagen immobilized interface. AFM studies of the sensor bio-interface confirms an expected minor deposition of platelets, (Figure $3 b$ ).

\section{Conclusions}

We demonstrated a novel concept of haemostasis assessment with the microacoustic sensor platform analyzing the platelets-collagen interaction at the biointerface. It is intended to improve the pathology evaluation in order to manage patients with bleeding risk or under antiplatelets therapy. In particular, the work is focused on the in-flow analysis of platelets-collagen interactions at the rheological conditions of the whole blood in-vivo. The initial results demonstrate the ability of selective platelets detection with a bulk acoustic wave resonator having a collagen immobilized biointerface. The obtained results are a first step towards the realization of an integrated microfluidic sensor solution for the study of collagen-platelets interactions in particular and assessment of primary haemostasis in general.

Author Contributions: A.O. developed microresonant sensor concept, completed the numerical study, performed experimental investigations with B.L.R.d.B, F.R.-M, A.R. and J.-F.M. and wrote the paper together with all co-authors. Bio-medical aspects of current work were covered by B.L.R.B, A.R. and W.B. Microtechnological approaches for designed sensor are contributed by F.C. Measurement software development, J.-F.M. Conceptualization, T.L., J.-F.M. Project Administration is completed by J.-F.M. and T.L. Funding Acquisition, J.-F.M. and T.L.

Funding: This research was funded by ANR GHOST No ANR-17-CE19-0026-01, the Interreg V France-Suisse 2014-2020 BLOODE convention No 1792 and the EUR EIPHI ANR-17-EUR-0002. This work was partly supported by the French RENATECH network and its FEMTO-ST technological facility.

Acknowledgments: Authors would like to thank G. Mourey from EFS (Etablissement français du sang) for the supply of the PPP, PRP and the whole blood samples, T. Lecompte from the Faculty of medicine of the University of Genova, Switzerland for his expertise and E. De Maistre from CHRU of Dijon whom provided the collagen Horm $^{\circledR}$ utilized in the experiments.

Conflicts of Interest: The authors declare no conflict of interest.

\section{References}

1. McCarty, O.J.T.; Ku, D.; Sugimoto, M.; King, M.R.; Cosemans, J.M.E.M.; Neeves, K.B. Dimensional analysis and scaling relevant to flow models of thrombus formation: Communication from the SSC of the ISTH. J. Thromb. Haemost. 2016, 14, 619-622, doi:10.1111/jth.13241.

2. Paniccia, R.; Priora, R.; Liotta, A.A.; Abbate, R. Platelet function tests: A comparative review. Vasc. Health Risk Manag. 2015, 11, 133, doi:10.2147/VHRM.S44469.

3. Weber, J.; Albers, W.M.; Tuppurainen, J.; Link, M.; Gabl, R.; Wersing, W.; Schreiter, M. Shear mode FBARs as highly sensitive liquid biosensors. Sens. Actuators A Phys. 2006, 128, 84-88, doi:10.1016/j.sna.2006.01.005.

4. Leblois, T.G.; Tellier, C.R.; Bourquin, R. The quality factor of deeply etched quartz resonators: Theory and experiments. Rev. Phys. Appl. 1989, 24, 877-892, doi:10.1051/rphysap:01989002409087700.

5. Kanazawa, K.K.; Gordon, J.G., II. The oscillation frequency of a quartz resonator in contact with liquid. Anal. Chim. Acta 1985, 175, 99-105, doi:10.1016/S0003-2670(00)82721-X. 
6. Sauerbrey, G.Z. The use of quartz oscillators for weighing thin layers and for microweighing. Z. Phys. 1959, 155, 206-222, doi:10.1007/BF01337937.

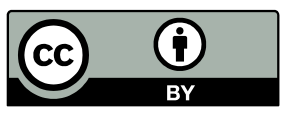

(C) 2018 by the authors. Licensee MDPI, Basel, Switzerland. This article is an open access article distributed under the terms and conditions of the Creative Commons Attribution (CC BY) license (http://creativecommons.org/licenses/by/4.0/). 